\title{
Breeding practices and mating strategies of Sheko cattle in south western Ethiopia
}

\author{
Takele Taye Desta $^{1 \dagger}$, Workneh Ayalew ${ }^{2}$ and B. P. Hegde ${ }^{3}$ \\ ${ }^{1}$ Wolayta Soddo Agricultural Technical Vocational Education and Training College, P.O. Box 120, Wolayta Soddo, Ethiopia; ${ }^{2}$ International Livestock Research Institute, \\ P.O. Box 5689, Addis Ababa, Ethiopia; ${ }^{3}$ Haramaya University, P.O. Box 138, Dire Dawa, Ethiopia
}

\begin{abstract}
Introduction
Sheko cattle owners usually have limited control over breeding practice of their cattle. This is because they allot fractions of their time and resources in controlling breeding practices. Therefore, most often mating is natural and uncontrolled and this would result in non-descript herd structure. Smallholder farmers usually practice frequent destocking and restocking of herds; therefore, this frequent herd turnover usually makes the tracing back of the pedigree structure of the herd impractical. Cattle are kept to fulfil multiple functions and, therefore, they are often not best in any one of the traits of interest. Cattle breeds kept by smallholder farmers show appreciable genetic variation in their performance. Therefore, there is room for within breed selection of indigenous breeds to improve their performance. However, this potential of local breeds under a low input production environment is not well documented. Our study was, therefore, intended to document cattle breeding practices of Sheko cattle owners in south western Ethiopia.
\end{abstract}

\section{Materials and methods}

Bench Maji Zone (BMZ), the main natural breeding tract of Sheko cattle, is located in the humid agro-ecological zone of south western Ethiopia. It lies between 850 and 3000 meters above sea level. The soil is mostly red brown with scattered tracts of red color. The annual temperature and rainfall range from 20 to $40^{\circ} \mathrm{C}$ and 1200 to $2000 \mathrm{~mm}$, respectively. BMZ receives maximum rain from June to September. The breeding tract is known for its mountain rainforests and is among the cradles of the origin of the highland forest coffee (Coffea arabica L.). Our study was conducted in sampled three districts of BMZ: Sheko, Bench and Shei Bench. A total of 35 purposively sampled villages were represented by randomly selected and individually interviewed 129 Sheko cattle owners. As an entry point during each interview, farmers were briefed about the objective of the study that followed by a brief open discussion. The pre-tested semi-structured questionnaire at 3 sites was administered to 51 and 78 Sheko cattle owners by trained enumerators in the lowland and highland area, respectively. This was supported by focus group discussions held at 10 villages and a number of key informant contacts and way-side informal talks.

\section{Results and discussions}

Cows dominate (61.9\%) herds of Sheko cattle and a similar finding was reported by Mwacharo and Drucker (2005) in Kenya. This herd structure showed a critical shortage of replacement breeding stock in both sex groups. Moreover, there is clear imbalance between female and male populations that worsen the shortage of breeding bulls. Reported sources of Sheko cattle fell into three categories: $53.5 \%$ home-born, $42 \%$ purchased and $4.5 \%$ sourced from absentee owners that entitled the herd attendant to use half of the milk. Reported sources for breeding bulls were $40.9 \%$ neighbors' herd, $31.8 \%$ own herd and $27.3 \%$ from communal grazing land. Reported selection criteria by the responders for breeding females were big udder and teat, high milk yield and healthy teats. Related conformation traits include wide hindquarter, long and thin tail, wide naval flap, thin and long neck, concave face, small hump, attractive look, drooping vulva (for calving ease), thick skin (to incapacitate biting flies attack) and large size. Other relevant traits include docile temperament, non-black hair coat, fast growth rate, good mothering ability and health status. Selection criteria used for bulls were alertness, which can be evaluated by the response of the animal. Moreover, strong stature, wide and well developed front quarter that narrows towards the hindquarter, short tail and thick neck reported as good conformation qualities of draught oxen. Large body size, long tail, thick skin, big hump, slowly emerging horns (early emergence of the horn believed to correlate with stunted growth) and wide dewlap. Other criteria include non-black coat color, attractive look, blocky appearance and fast growth, having normal testis and big scrotum. Out of the total responders $92.3 \%$ reported the practice of bull castration. The reported reasons for non-practitioners were having no bulls, sale of bull calves at earlier age, keeping bulls longer for breeding; and few responders reported that their cows entirely gave birth to female calves. The reported reasons for castration were for good finish (55.9\%), to control aggressive behavior (40.6) and to prevent weight loss (3.5\%, $N=170$ ). Year round calving was reported by $91.4 \%$ of the responders, whereas $8.6 \%$ of them reported that calving is slightly higher in some seasons of the year. This was mainly due to seasonal feed availability which was reported to affect the libido of the bulls and fertility of the cows. Mating is natural and $45 \%$ of the responders reported selection of bulls that have brighter colors and large size upon mating (Taye, 2005).

\section{Conclusions}

There are opportunities to improve the performance of Sheko cattle by selection. Smallholder farmers have their own breeding strategies to cope with their production environment while fulfilling their needs for subsistence. Therefore, their indigenous knowledge should be considered in planning and implementation of smallholder cattle breeding programs.

\footnotetext{
† E-mail: takele_taye@yahoo.com
} 


\author{
References \\ Taye T 2005. MSc thesis, Haramaya University. 105+xiv. \\ Mwacharo JM and Drucker AG 2005. Tropical Animal Health and Production 37, 635-652.
}

doi:10.1017/S2040470010000221

\title{
Phenotypic characterization of the local goat in Mauritius
}

\author{
Saraye Geerjanand ${ }^{\dagger}$
}

Agricultural Research and Extension Unit, Newry complex, 230 Quatre Bornes, Mauritius

Introduction

Goat rearing has always been an important activity for meat production in the rural and sub-urban areas of Mauritius, representing an additional source of revenue and a cash reserve for many farmers. In 2005 there were about 16500 heads of goats and 2500 goat farmers in the country (Anon, 2005). Most of the goats are kept in small units of 5-10 heads under backyard production systems whereby the animals are confined to their sheds and fodder is provided on a cut-and-carry basis. However, there are also many farms where the flock size varies from 10 to 25 and a few with flocks exceeding 25 heads.

Goats were first introduced into Mauritius in 1606, when a Dutch admiral is reported to have left 24 goats on the island (Lestrac (de) J.C, 2007). The local goats are descendants of this first population of goats and have for many centuries been subjected to the particular local environmental conditions. As a result, they have developed certain valuable genetic traits such as ability to perform under low input condition and climatic stress and resistance to infectious diseases and parasitism. The local breed was regarded as less productive and therefore subjected to replacement and cross-breeding with imported breeds like British Alpine, Anglo Nubian, Jumna Pari and Boer introduced at different periods since 1950. This has resulted in a drastic reduction in the number of local goats (breed substitution) and the loss of their characteristics (genetic erosion). The genetic purity of the local breed is now under severe threat due to indiscriminate adoption of exotic breeds.

This study therefore aimed to phenotypically characterise the local goat in view of establishing a database for the breed for use in future upgrading and breeding programmes.

\section{Materials and methods}

Traditional goat farms (117) were randomly selected and surveyed islandwide in 2005. Records on morphological characteristics were taken on 347 (151 males and 196 females) adult animals randomly selected on the farms. Traits scored were presence or absence of horns, shape of horns, orientation of horns, presence or absence of wattles, types of ears, color of coat, texture of coat, face profile, rump profile, back profile, orientation of ears, coat type, score of hair length and presence or absence of beard for each animal. The sex of each animal was also recorded.

\section{Results}

The study showed that mature males averaged $57.9 \pm 5.5 \mathrm{~cm}$ at the shoulder, had an average chest-girth of $68.9 \pm 7.2 \mathrm{~cm}$, an average body length of $58.2 \pm 8.7 \mathrm{~cm}$ and an average body weight of $25.2 \pm 5.1 \mathrm{~kg}$. In mature females shoulder height averaged $54.6 \pm 5.0 \mathrm{~cm}$, chest-girth $66.7 \pm 6.5 \mathrm{~cm}$, body length $56.0 \pm 6.3 \mathrm{~cm}$ and body weight $23.9 \pm 5.5 \mathrm{~kg}$. Most of the animals, $61.7 \%$ were of uniform color; black, being the most common of goats, $48.7 \%$, followed by $6.9 \%$ white and $6.1 \%$ brown. The animals $(68.3 \%)$ showed mixed or other combinations of colors in varying proportions. Hairs were generally short and straight. Less than $2 \%$ of animals had short curly or long straight hair. The facial profile was predominantly straight (flat) with only $4.0 \%$ of the animals having convex or concave profiles. Ears were carried laterally in $85.0 \%$ and horizontally forward in $3.2 \%$ of the animals. The animals $(4.3 \%$ and $6.1 \%)$ had erect and pendulous ears respectively. Ears averaged $13.4 \pm 2.0 \mathrm{~cm}$ in length. Males (59.9\%) carried beards as compared to only $12.8 \%$ of females. Horns were present in $95.4 \%$ of animals but $7.0 \%$ had only rudimentary horns or scurs. The horns generally grew in a backward curve (45.2\%) or straight backwards (18.2\%). Animals $(48.1 \%)$ had long horns $(>8 \mathrm{~cm}), 44.9 \%$ had horns of medium length $(2-8 \mathrm{~cm})$ and $7.1 \%$ had short horns $(<2 \mathrm{~cm})$.

\section{Conclusion}

The local goat can be characterized as a small to medium size (Galal, 2007) breed with variable coat color and pattern, short straight hair, straight facial profile, medium size laterally carried ears and predominantly horned. Since the breed has been acclimatized to the local environment there is an urgent need to conserve and use it in a breeding programme.

\footnotetext{
†E-mail: nimishe@yahoo.com
} 